\title{
ORIGINAL
}

\section{CALIDAD DE VIDA RELACIONADA CON LA SALUD Y HÁBITOS DE VIDA: DIFERENCIAS ENTRE ADOLESCENTES MIGRANTES Y AUTÓCTONOS(*)}

\author{
Raúl Jiménez Boraita (1), Daniel Arriscado Alsina (1), Josep María Dalmau Torres (1) y Esther Gargallo Ibort (1)
}

(1) Departamento de Ciencias de la Educación. Universidad de La Rioja. Logroño. España.

Los autores declaran que no existe conflicto de intereses.

(*) Financiación: El estudio fue parcialmente financiado por el Instituto de Estudios Riojanos (IER) del Gobierno de La Rioja a través de la "Resolución n ${ }^{\circ} 55 / 2018$, de 9 de julio, de la Gerencia del Instituto de Estudios Riojanos para la concesión de ayudas para estudios científicos de temática riojana convocadas para el año 2018-2019".

\section{RESUMEN}

Fundamentos: Los flujos migratorios han provocado que la población migrante se convierta en un colectivo importante en la sociedad española, haciendo necesaria su integración tanto en el ámbito social como escolar. Con ese propósito, se analizaron las diferencias entre migrantes y autóctonos en una población de adolescentes de una región del norte de España desde una perspectiva integral de la salud, evaluando distintos indicadores tanto físicos como psicológicos y sociales.

Métodos: Estudio trasversal aplicado a una muestra de 761 escolares, distribuidos en 618 autóctonos $(14,49$ $\pm 1,62$ años) y 143 migrantes (14,55 $\pm 1,66$ años). Se realizó un análisis descriptivo, comparativo y correlacional de los datos obtenidos de las variables de adherencia a la dieta mediterránea, nivel de actividad física, calidad de vida relacionada con la salud, autoestima, satisfacción de la imagen corporal, horas de sueño nocturno, índice de masa corporal, consumo máximo de oxígeno, rendimiento académico y nivel socioeconómico.

Resultados: El alumnado migrante presentó valores significativamente menores en el nivel socioeconómico $(p<0,001)$, rendimiento académico $(p<0,001)$, autoestima $(\mathrm{p}<0,05)$, nivel de actividad física $(\mathrm{p}<0,05)$, capacidad aeróbica $(p<0,05)$, adherencia a la dieta mediterránea $(\mathrm{p}<0,05)$ y satisfacción corporal $(\mathrm{p}<0,05)$. En relación a la calidad de vida relacionada con la salud $(p<0,001)$, se reportaron diferencias a nivel global y en todas las dimensiones analizadas, excepto en la satisfacción con el entorno educativo. Además, exclusivamente en el caso de los migrantes, el nivel socioeconómico se asoció con el apoyo social $(\mathrm{r}=0,256)$, el rendimiento académico $(\mathrm{r}=0,261)$ y la adherencia a la dieta mediterránea $(\mathrm{r}=0,166)$.

Conclusiones: Los resultados obtenidos destacan el relevante papel que el contexto escolar ejerce como promotor de salud e integración en los alumnos migrantes. Se hace necesaria la implementación de estrategias que promuevan el acceso a la práctica física y a alimentos saludables con el objetivo de favorecer la mejora de la salud comunitaria y, en especial, de los grupos más vulnerables.

Palabras clave: Migrantes, Estilo de vida, Calidad de vida, Bienestar físico, Bienestar psicológico.

\section{ABSTRACT \\ Health-related quality of life and lifestyle habits: differences between migrant and native adolescents}

Background: The migratory flows have caused the migrant population to become an important collective of the Spanish social reality, being necessary to know their situation to favor their integration, both in the social and school environment. With this purpose, the differences between a population of migrant and native adolescents from a region of northern Spain were analyzed from an integral perspective of health, evaluating different indicators of physical, psychological and social health.

Methods: Cross-sectional study was applied to a sample of 761 schoolchildren, distributed in 618 native (14.49 \pm 1.62 years) and 143 migrants $(14.55 \pm 1.66$ years). A descriptive, comparative and correlational analysis was performed of the data obtained from the variables of adherence to the Mediterranean diet, level of physical activity, health-related quality of life, selfesteem, body image satisfaction, hours of nighttime sleep, index body mass, maximum oxygen consumption, academic performance and socioeconomic status.

Results: Migrant students presented significantly lower values in socioeconomic status $(p<0.001)$, academic performance $(p<0.001)$, self-esteem $(p<0.05)$, level of physical activity $(\mathrm{p}<0.05)$, aerobic capacity $(p<0.05)$, adherence to the Mediterranean $\operatorname{diet}(p<0.05)$ and body satisfaction $(\mathrm{p}<0.05)$. Regarding the quality of life related to health, differences were reported in the global $(p<0.001)$ and in all the dimensions analyzed except in satisfaction with the educational environment. In addition, exclusively in the case of migrants, the socioeconomic status was associated with social support $(\mathrm{r}=0.256)$, academic performance $(\mathrm{r}=0.261)$ and adherence to the Mediterranean diet $(\mathrm{r}=0.166)$.

Conclusions: The results obtained highlight the relevant role that the school context plays as a health and integration promoter in migrant students. It is necessary to implement strategies that promote access to physical practice and healthy food with the objective of promoting the improvement of community health and, especially, of the most vulnerable groups.

Key words: Migrants, Lifestyle, Quality of life, Physical well-being, Psychological well-being.

Cita sugerida: Jiménez Boraita $\mathrm{R}$, Arriscado Alsina D, Dalmau Torres JM, Gargallo Ibort E. Calidad de vida relacionada con la salud y hábitos de vida: diferencias entre adolescentes migrantes y autóctonos. Rev Esp Salud Pública. 2020; 94: 13 de abril e202004023
Correspondencia:

Raúl Jiménez Boraita

Avenida Fuenmayor, $\mathrm{n}^{\circ} 5,1^{\circ} \mathrm{D}$

26350 Cenicero, España

rauljbcity@gmail.com 


\section{INTRODUCCIÓN}

El volumen de movimientos migratorios acontecidos en las últimas décadas ha consolidado a España como uno de los mayores países receptores de migrantes del continente europeo. Esto ha originado una importante transformación en la sociedad, siendo más heterogénea en la actualidad que en tiempos pasados. Atendiendo a los datos recogidos en el Padrón Continuo de España, elaborado por el Instituto Nacional de Estadística, la población extranjera representaba el $10,7 \%$ de la población total española a 1 de enero de $2019^{(1)}$. Si reducimos el espectro al grupo de edad entre 10 y 19 años, el porcentaje de representatividad migrante era del $8,74 \%$.

Esta nueva realidad social, derivada de los citados cambios poblacionales, ha suscitado el interés por comprender e integrar a la población migrante en la comunidad, siendo la salud y la educación dos de los ámbitos de mayor atención ${ }^{(2)}$. Los diferentes condicionantes de vida ambientales, económicos y genéticos, además de los factores sociales, pueden definir en gran medida el estado de salud de la población ${ }^{(3,4)}$. Asimismo, los hábitos de vida también parecen ejercer un papel clave en la salud adolescente ${ }^{(5)}$, destacando la relación hallada en estudios anteriores entre los hábitos de vida y la mortalidad $^{(6)}$, e indicando que aquellos hombres y mujeres que tenían hábitos de vida saludables reducían el porcentaje de mortandad en un $28 \%$ y $43 \%$, respectivamente, en relación a quienes no los tenían. Por ello, resulta importante considerar que la población adolescente, tanto migrante como autóctona, se encuentra en pleno proceso de adquisición de hábitos que definirán en gran medida sus estilos de vida durante la edad adulta ${ }^{(7)}$.

Los escasos estudios que comparan el estado de salud de la población migrante y la autóctona reflejan diferencias significativas entre ambas poblaciones. En este sentido, las condiciones de vida de la población migrante pueden estar definidas por el propio proceso migratorio, en el que la adaptación al nuevo entorno, la vulnerabilidad social y el nivel socioeconómico determinan su estado de salud ${ }^{(8)}$. En este sentido, la diferencia de oportunidades generada por estas barreras económicas y sociales puede inducir a la adquisición de hábitos no saludables, como pueden ser una menor realización de actividad física $(\mathrm{AF})^{(9)}$ o el consumo de alimentos poco recomendables ${ }^{(10)}$, además de ocasionar un mayor riesgo de padecer diferentes problemas psicológicos y de adaptación al entorno ${ }^{(11)}$. Las citadas diferencias tienden a reducirse cuando los condicionantes económicos, la adaptación social y el tiempo de residencia se van igualando entre migrantes y autóctonos ${ }^{(12)}$. Por otro lado, también cabe destacar el importante papel integrador que los contextos escolares ofrecen a la población migrante, proporcionando a estudiantes y familias un entorno favorable para el contacto con la cultura y sociedad del país receptor ${ }^{(13)}$.

La generalidad de estos estudios se ha centrado en un análisis parcial de los componentes de salubridad, siendo escasas las investigaciones que aporten resultados desde un enfoque holístico de la salud. De este modo, el objeto del presente trabajo fue analizar las diferencias entre una población de adolescentes tanto migrantes como nacionales de una región del norte de España desde una perspectiva integral de la salud, evaluando para ello sus hábitos de vida, así como diferentes indicadores de su salud, tanto física como psicológica y social. A tal fin, se valoró el nivel de $\mathrm{AF}$, el consumo máximo de oxígeno $\left(\mathrm{VO}_{2}\right.$ máx), las horas de sueño nocturno, la calidad de vida relacionada con la salud (CVRS), la autoestima, la percepción y satisfacción de la imagen corporal, la adherencia a la dieta mediterránea (DM), el índice de masa corporal (IMC), el rendimiento académico y el nivel socioeconómico. 


\section{SUJETOS Y MÉTODOS}

Se propuso un estudio descriptivo, transversal y correlacional en el contexto socioeducativo. La muestra se obtuvo mediante un muestreo aleatorio estratificado por conglomerados, considerando como unidad muestral las aulas de primer y cuarto curso de Educación Secundaria de todos los centros de la Comunidad Autónoma de La Rioja. Se asumió un error del 5\% y un nivel de confianza del $95 \%$, conformando una muestra total de 761 adolescentes $(14,51 \pm 1,63$ años) procedentes de 25 centros educativos. De este modo, el $81,2 \%$ de la muestra estaba representada por los autóctonos, entre los que se incluyó a quienes habían nacido en España y tenían al menos uno de sus progenitores también nacido en España. El 18,8\% de la muestra fue compuesta por los adolescentes migrantes, grupo conformado por los migrantes de primera generación (sin nacionalidad española) y por los de segunda generación (con nacionalidad española pero con ambos progenitores migrantes). La inclusión de estos últimos en el grupo de migrantes se justificó mediante un análisis previo en el que no se encontraron diferencias significativas entre ambos grupos.

Se respetaron los fundamentos de ética de investigación de Helsinki. Además, se requirió el consentimiento informado por parte de los progenitores o tutores legales. El proyecto fue aprobado por el Comité Ético de Investigación Clínica de La Rioja. Las variables analizadas en el estudio se detallan a continuación:

Autoestima. Se evaluó con la escala de autoestima de Rosenberg, validada y adaptada para la población adolescente española por Atienza et $\mathrm{al}^{(14)}$. La escala está formada por diez ítems que se puntúan del uno al cuatro en función a sus respuestas y que dan lugar a una valoración global entre diez y cuarenta. Los valores más altos representan una mayor autoestima.
Calidad de vida relacionada con la salud (CVRS). Fue estimada con el cuestionario KIDSCREEN 27, validado para adolescentes españoles por Aymerich et al ${ }^{(15)}$. Está formado por 27 ítems de tipo Likert, agrupados en cinco dimensiones: bienestar físico; bienestar psicológico; autonomía y padres; entorno escolar; apoyo social y amigos. Los valores más altos representan una mayor percepción de CVRS.

Percepción y satisfacción de la imagen corporal. Se valoró utilizando el método Stunkard y Stellar ${ }^{(16)}$. Se evaluó a través de nueve figuras masculinas y femeninas, que fueron progresivamente más robustas en apariencia y se etiquetaron con un IMC valorado entre uno (IMC 17 $\mathrm{kg} / \mathrm{m}^{2}$ ) y nueve (IMC $33 \mathrm{~kg} / \mathrm{m}^{2}$ ). Los participantes escogieron la silueta que más se asemejaba a su apariencia y la que deseaban tener. El IMC asociado con la silueta deseada se resta al IMC asociado con la silueta percibida, obteniendo valores que se categorizan según lo descrito en la adaptación al español de Marrodán et al ${ }^{(17)}$.

Adherencia a la dieta mediterránea (DM). Para valorarla se utilizó el cuestionario Mediterranean Diet Quality (KIDMED) diseñado por Serra et al ${ }^{(18)}$. Consta de dieciséis ítems de repuesta dicotómica que afirman o niegan el consumo de una serie de alimentos identitarios de la dieta mediterránea. Del cuestionario se obtiene una puntuación entre menos cuatro y doce, asociándose los valores más altos a una mayor adherencia.

Nivel de actividad física (AF). Se estimó con el cuestionario Physical Activity Questionnaire for Adolescents (PAQ-A), adaptado para adolescentes españoles por Martínez et al ${ }^{(19)}$. Se valoró la AF realizada según frecuencias y franjas horarias durante la última semana. Los valores obtenidos se encuentran entre el uno y el cinco, estando asociados los mayores niveles de AF a las puntuaciones más altas. También 
se incorporaron preguntas referentes a las horas de sueño nocturno, la realización de actividades deportivas organizadas en horario extraescolar y el medio de transporte utilizado para el desplazamiento hasta el centro educativo.

Además, con el propósito de detectar y eliminar del análisis los cuestionarios que fueron respondidos de manera aleatoria, deshonesta o pseudoaleatoria, se incluyeron seis ítems de la Escala de Frecuencia de Respuesta de Oviedo $(\mathrm{INF}-\mathrm{OV})^{(20)}$ de forma intercalada a lo largo del cuestionario. El INF-OV es un instrumento de autoinforme de respuestas dicotómicas donde se refleja el grado de acuerdo $(0=$ de acuerdo, $1=$ en desacuerdo). La puntuación se obtiene de la suma de las 6 preguntas. Aquellos que obtuvieron más de un punto fueron eliminados del análisis.

Capacidad aeróbica. Para su evaluación se realizó el Test Course-Navette. Para ello, se fijaron unas marcas trasversales separadas por una distancia de 20 metros que señalaban el principio y fin de un recorrido por el que los participantes debían desplazarse, según un ritmo inicial de carrera de $8,5 \mathrm{~km} / \mathrm{h}$ que se incrementaba $0,5 \mathrm{~km} / \mathrm{h}$ cada minuto, y que era señalado por un indicador acústico. La prueba finaliza cuando el participante no logra completar por segunda vez consecutiva la distancia entre las dos líneas en el ritmo marcado o cuando se retira. Con los resultados obtenidos se estimó el VO2 máximo según las fórmulas determinadas por el autor del test ${ }^{(21)}$.

Índice de masa corporal. El peso se midió con una balanza SECA ${ }^{\circledR}$ (modelo 713, Hamburgo, Alemania) con una precisión de $0,1 \mathrm{Kg}$. La talla se registró con un tallímetro Holtain ${ }^{\circledR}$ (Holtain Ltd., Dyfed, Reino Unido) con una precisión de un milímetro. Una vez obtenidas las medidas, se calculó el índice de masa corporal (IMC) y se categorizó según las referencias establecidas por la $\mathrm{OMS}^{(22)}$, diferenciando entre "Normopeso", "Sobrepeso" y "Obesidad".
Nivel socioeconómico. Para su evaluación se utilizó el Family Affluence Scale (FAS) ${ }^{(23)}$, que determina este nivel en función de seis preguntas relacionadas con la posesión de bienes materiales familiares. De las respuestas se obtiene una puntuación entre cero y trece, siendo cero el poder adquisitivo más bajo y trece el más alto.

Calificaciones académicas. Fueron proporcionadas por parte de la Consejería de Educación del Gobierno de La Rioja, tras obtener un consentimiento específico por parte de las familias. Las calificaciones representaban la nota media obtenida por el alumnado en el curso en que se encontraba al participar en el estudio. Un total de 684 familias accedieron a la cesión de las calificaciones de sus hijos, distribuidas en 123 por parte de los migrantes $(86,01 \%)$ y 561 de los autóctonos (90,77\%).

Con todos los datos recogidos, se realizó un análisis estadístico descriptivo, mostrando los resultados de las variables cuantitativas mediante sus medias y desviaciones típicas, y de las variables cualitativas mediante las frecuencias. La normalidad de los datos se constató con el test de Kolmogorov-Smirnov, con corrección de la significación de Lilliefors, y la de homocedasticidad con el test de Levene. La comparación de medias entre migrantes y autóctonos se realizó con la prueba de $\mathrm{T}$ de Student y $\mathrm{U}$ de Mann-Whitney según su normalidad. Con el test Chi-cuadrado de Pearson se analizó la asociación de las variables cualitativas. El análisis de asociación entre variables se llevó a cabo mediante el índice de correlación de Pearson y el de Spearman, para las distribuciones normales y no normales, respectivamente. Se realizó un análisis de regresión simple con el objetivo de determinar la influencia entre algunas de las variables estudiadas. Para el análisis estadístico se utilizó el programa IBM-SPSS ${ }^{\circledR}$, versión 25 , estableciendo un nivel de significación de $\mathrm{p}<0,05$. 


\section{RESULTADOS}

En la tabla 1 se pueden observar los resultados obtenidos en relación al nivel socioeconómico, $\mathrm{AF}$ y $\mathrm{VO}_{2}$ máx, CVRS, autoestima, horas de sueño, DM, IMC y rendimiento académico en función de la nacionalidad. Todas las variables descritas presentaron valores significativamente inferiores por parte de los migrantes, excepto en horas de sueño (donde no se encontraron diferencias) e IMC (donde los migrantes presentaron valores significativamente superiores). En relación al nivel socioeconómico, destacar que este se asoció exclusivamente con el rendimiento académico en los adolescentes migrantes $(\mathrm{r}=0,261 ; \mathrm{p}<0,05)$, explicando el $6,4 \%$ de la varianza de este por solo el $1,1 \%$ de la de los autóctonos.

La tabla 2 recoge los resultados de la CVRS y sus cinco dimensiones. Los adolescentes migrantes presentaron valores significativamente

\begin{tabular}{|c|c|c|c|c|c|}
\hline \multicolumn{6}{|c|}{$\begin{array}{c}\text { Tabla } 1 \\
\text { Características de la muestra en función de la nacionalidad. }\end{array}$} \\
\hline \multirow{2}{*}{ Variables } & \multicolumn{2}{|c|}{ Autóctonos $(\mathrm{n}=618)$} & \multicolumn{2}{|c|}{ Migrantes $(n=143)$} & \multirow{2}{*}{$p$ valor } \\
\hline & M & $\sigma$ & M & $\sigma$ & \\
\hline Nivel Socioeconómico & 9,41 & 0,07 & 7,33 & 0,19 & 0,0001 \\
\hline AF (PAQ-A) & 2,63 & 0,02 & 2,49 & 0,05 & 0,005 \\
\hline $\mathrm{VO}_{2}$ máx & 44,66 & 0,27 & 42,45 & 0,59 & 0,001 \\
\hline CVRS (Kidscreen) & 252,27 & 1,29 & 240,95 & 3,01 & 0,0001 \\
\hline Autoestima & 32,89 & 0,19 & 31,83 & 0,43 & 0,019 \\
\hline Horas de sueño nocturno & 8,36 & 0,35 & 8,36 & 0,08 & 0,320 \\
\hline DM (Kidmed) & 7,41 & 0,08 & 6,85 & 0,19 & 0,027 \\
\hline IMC & 20,78 & 0,12 & 21,99 & 0,32 & 0,002 \\
\hline Rendimiento académico & 6,76 & 0,05 & 5,79 & 0,14 & 0,0001 \\
\hline
\end{tabular}

\begin{tabular}{|c|c|c|c|c|c|}
\hline \multicolumn{6}{|c|}{$\begin{array}{c}\text { Tabla } 2 \\
\text { Calidad de vida en función de la nacionalidad. }\end{array}$} \\
\hline \multirow{2}{*}{ Variables } & \multicolumn{2}{|c|}{ Autóctonos $(\mathrm{n}=618)$} & \multicolumn{2}{|c|}{ Migrantes $(n=143)$} & \multirow{2}{*}{$p$ valor } \\
\hline & M & $\sigma$ & M & $\sigma$ & \\
\hline CVRS (Kidscreen) & 252,27 & 1,29 & 240,95 & 3,01 & 0,0001 \\
\hline Bienestar Físico & 45,86 & 0,34 & 44,35 & 0,78 & 0,038 \\
\hline Bienestar Psicológico & 50,54 & 0,37 & 48,51 & 0,8 & 0,005 \\
\hline Entorno Educativo & 49,94 & 0,36 & 48,56 & 0,86 & 0,108 \\
\hline Autonomía y Padres & 51,67 & 0,34 & 48,74 & 0,73 & 0,0001 \\
\hline Apoyo Social y Amigos & 54,25 & 0,36 & 50,57 & 0,82 & 0,0001 \\
\hline
\end{tabular}


inferiores $(\mathrm{p}<0,001)$ en el cómputo global de CVRS. Además, en relación a las diferentes dimensiones de las que consta el cuestionario, los migrantes mostraron niveles significativamente más bajos $(p<0,05)$ en todas ellas excepto en el entorno educativo, donde no se hallaron diferencias significativas. En cuanto al apoyo social, los migrantes reportaron una asociación directa entre el mismo y el nivel socioeconómico $(\mathrm{r}=0,256 ; \mathrm{p}<0,01)$.

El nivel de AF global y también durante los diferentes momentos del día y de la semana se muestran en la tabla 3 . Los adolescentes migrantes presentaron valores significativamente más bajos que el alumnado autóctono en el global del cuestionario $(p<0,05)$. En relación a las franjas horarias, los migrantes presentaron menores niveles de AF $(\mathrm{p} \leq 0,001)$ entre las 18:00 y las 22:00 horas. También fueron menores la intensidad semanal y la frecuencia diaria. No se hallaron diferencias en el tramo horario escolar, ni en las clases de Educación Física ni en el recreo. Además, mostraron índices inferiores de participación en actividades deportivas extraescolares $(\mathrm{p}<0,001)$, presentando un porcentaje del $48,3 \%$ frente a un $75,1 \%$ en autóctonos.

Los resultados obtenidos en relación a la DM y al consumo de alimentos asociados a ella se recogen en la tabla 4. La adherencia fue mayor por parte de los adolescentes autóctonos $(\mathrm{p}<0,05)$. En relación al consumo de alimentos concretos, se encontró una menor ingesta $(p<0,01)$ de pescado, legumbres, aceite de oliva y lácteos por parte de los migrantes. Por el contrario, reportaron un mayor consumo $(\mathrm{p} \leq 0,01)$ de comida rápida, pasta, arroz y dulces. El estudio correlacional advirtió una asociación positiva entre la DM y el nivel socioeconómico $(\mathrm{r}=0,166 ; \mathrm{p}<0,01)$, que explicaba el $3,1 \%$ de la varianza en los migrantes y el $0,16 \%$ en los autóctonos.

Por último, en la tabla 5 se representan los resultados obtenidos en relación al IMC. Se revelaron diferencias significativas $(\mathrm{p}<0,001)$ en la composición corporal, destacando los

\begin{tabular}{|l|c|c|c|c|c|}
\hline \multicolumn{7}{|c|}{ Tabla 3 } \\
\multicolumn{2}{|c|}{ Nivel de actividad física en función de la nacionalidad. } \\
\cline { 1 - 6 } & Variables & Autóctonos $(\mathrm{n}=618)$ & \multicolumn{2}{c|}{ Migrantes $(\mathrm{n}=143)$} & \multirow{2}{*}{$p$ valor } \\
\cline { 2 - 6 } & $\mathrm{M}$ & $\sigma$ & $\mathrm{M}$ & $\sigma$ & \\
\hline Nivel de AF (PAQ-A) & 2,63 & 0,02 & 2,49 & 0,05 & 0,005 \\
\hline Lista de actividades semanales & 1,43 & 0,01 & 1,41 & 0,02 & 0,611 \\
\hline Educación Física & 3,98 & 0,36 & 3,98 & 0,07 & 0,733 \\
\hline Recreo & 2,2 & 0,04 & 2,32 & 0,09 & 0,282 \\
\hline Comida & 1,48 & 0,03 & 1,57 & 0,07 & 0,316 \\
\hline Tarde (14-18h) & 2,75 & 0,04 & 2,64 & 0,1 & 0,282 \\
\hline Tarde (18-22h) & 3,05 & 0,04 & 2,64 & 0,1 & 0,0001 \\
\hline Fin de Semana & 2,69 & 0,04 & 2,51 & 0,89 & 0,057 \\
\hline Intensidad Semanal & 2,71 & 0,04 & 2,41 & 0,09 & 0,001 \\
\hline Frecuencia Diaria & 3 & 0,03 & 2,73 & 0,06 & 0,0001 \\
\hline
\end{tabular}




\section{Tabla 4}

Adherencia a la dieta mediterránea en función de la nacionalidad.

\begin{tabular}{|l|c|c|c|}
\hline \multicolumn{1}{|c|}{ Variables } & $\begin{array}{c}\text { Autóctonos } \\
(\mathrm{n}=618)\end{array}$ & $\begin{array}{c}\text { Migrantes } \\
(\mathrm{n}=143)\end{array}$ & \multirow{2}{*}{$p$ valor } \\
\cline { 2 - 3 } & $\%$ SÍ & $\%$ SÍ & \\
\hline Adherencia a DM (KidMed) & $7.41 \pm 0.08$ & $6.85 \pm 0.19$ & 0,027 \\
\hline Desayuno diario & $95 \%$ & $91,60 \%$ & 0,115 \\
\hline Lácteos en el desayuno & $88,80 \%$ & $79,70 \%$ & 0,003 \\
\hline Cereal o derivados en el desayuno & $80,40 \%$ & $82,50 \%$ & 0,566 \\
\hline Desayuno con bollería industrial en el desayuno & $20,40 \%$ & $18,90 \%$ & 0,685 \\
\hline Fruta o zumo de fruta natural de diario & $67,30 \%$ & $67,10 \%$ & 0,967 \\
\hline Segunda pieza de fruta de diario & $49,20 \%$ & $48,30 \%$ & 0,840 \\
\hline Segundo lácteo de diario & $76,70 \%$ & $55,90 \%$ & 0,0001 \\
\hline Verduras frescas (ensaladas) o cocinadas de diario & $71,70 \%$ & $77,60 \%$ & 0,150 \\
\hline Verduras frescas o cocinadas más de una vez al día & $31,60 \%$ & $37,80 \%$ & 0,154 \\
\hline Consumo de Pescado (al menos 2 o 3 veces a la semana) & $67,30 \%$ & $53,80 \%$ & 0,002 \\
\hline Consumo de frutos secos (al menos 2 o 3 veces a la semana) & $48,20 \%$ & $44,80 \%$ & 0,455 \\
\hline Consumo en centros de fast-food (una vez o más a la semana) & $17,80 \%$ & $27,30 \%$ & 0,01 \\
\hline Consumo de legumbres (más de una vez a la semana) & $87,10 \%$ & $71,30 \%$ & 0,0001 \\
\hline Consumo de pasta o arroz (más de cuatro veces a la semana) & $35,10 \%$ & $55,20 \%$ & 0,0001 \\
\hline Consumo de dulces o golosinas (varias veces al día) & $17,60 \%$ & $28,70 \%$ & 0,003 \\
\hline Uso aceite de oliva en casa & $98,40 \%$ & $94,40 \%$ & 0,005 \\
\hline
\end{tabular}

\section{Tabla 5}

Satisfacción corporal y composición corporal de la muestra según la clasificación de la OMS.

\begin{tabular}{|l|c|c|c|c|}
\hline \multicolumn{2}{|c|}{ Variables } & $\begin{array}{c}\text { Autóctonos } \\
(\mathrm{n}=618)\end{array}$ & $\begin{array}{c}\text { Migrantes } \\
(\mathrm{n}=143)\end{array}$ & \multirow{2}{*}{$p$ valor } \\
\hline \multirow{3}{*}{ Clasificación OMS } & Normopeso & $76,5 \%$ & $61,5 \%$ & \multirow{2}{*}{0,0001} \\
\cline { 2 - 4 } & Sobrepeso & $18,3 \%$ & $25,2 \%$ & \\
\cline { 2 - 4 } & Obesidad & $5,2 \%$ & $13,3 \%$ & \\
\hline \multirow{3}{*}{ IMC Real - Deseado } & Desea mayor IMC & $34,50 \%$ & $35 \%$ & \multirow{2}{*}{0,018} \\
\cline { 2 - 4 } & Conformidad & $47,20 \%$ & $37,10 \%$ & \multirow{2}{*}{$28 \%$} \\
\cline { 2 - 4 } & Desea menor IMC & $18,30 \%$ & $28 \%$ \\
\hline
\end{tabular}


mayores porcentajes por parte de los migrantes frente a los de los autóctonos en cuanto a sobrepeso y obesidad. En relación con la satisfacción corporal, el 28\% de los migrantes manifestaron un deseo de alcanzar un menor IMC, frente al 18,3\% de los autóctonos $(\mathrm{p}<0,05)$. El análisis de correlación mostró, en el caso de los migrantes, una asociación entre la insatisfacción corporal y el bienestar psicológico $(r=-0,165 ; \mathrm{p}<0,05)$.

\section{DISCUSIÓN}

La vulnerabilidad, el riesgo de exclusión y las condiciones de vida a las que está sometida la población migrante dificultan su estabilidad psicosocial y económica, redundando en su salud y bienestar de manera clave ${ }^{(24)}$. Nuestro análisis ratifica este hecho, mostrando diferencias entre el alumnado migrante y el nacional en todas las variables estudiadas, excepto en las horas de sueño.

Centrándonos en los valores obtenidos en la CVRS, los adolescentes autóctonos muestran valores significativamente superiores en lo que respecta a la relación con los padres y su autonomía, al apoyo social y al bienestar físico y psicológico. Esta situación se asocia al propio proceso de adaptación y a las condiciones de vida, donde los lazos socioculturales que los migrantes mantienen hacia su país de procedencia y las diferencias con el contexto social de acogida generan conflictos en el proceso de aculturación, observándose una mayor presión y necesidad de ajuste en el plano psicosocial ${ }^{(25)}$. Estas mismas razones podrían atribuirse a los menores índices de autoestima reportados por los alumnos migrantes.

En relación al apoyo social, los adolescentes migrantes presentan valores más bajos, además de una asociación entre el nivel socioeconómico y la relación con sus pares. De acuerdo con Hjalmarsson et $\mathrm{al}^{(26)}$, los alumnos de familias con menor poder económico presentan escasez de relaciones sociales en el aula debido a la carencia de recursos materiales propios o de oportunidades para realizar actividades grupales, lo que justificaría estos bajos resultados en el bienestar social.

Por el contrario, los resultados evidencian el papel protector que desempeña el centro educativo durante el proceso de adaptación y bienestar del alumnado migrante, puesto que en la dimensión referente a la satisfacción en el entorno escolar no se encuentran diferencias respecto al alumnado autóctono. Cabe señalar la necesidad de continuar trabajando por la equidad en la educación, así como ofrecer los medios y recursos para favorecer el desarrollo integral de todos los alumnos, tal y como contempla el marco legislativo español en el Titulo II de la Ley Orgánica para la Mejora de la Calidad Educativa ${ }^{(27)}$.

Esta necesidad se refleja igualmente al observar que los adolescentes migrantes reportan calificaciones más bajas, mostrándose en su caso una asociación entre el nivel socioeconómico y el rendimiento académico. Zinovyeva et $\mathrm{al}^{(28)}$ indicaron como factores predictivos de estas desigualdades diferentes condicionantes de vida como el ambiente cultural y familiar, el manejo del idioma, el nivel de formación académica de los progenitores y el nivel socioeconómico, destacando este último factor como clave de los contrastes entre el rendimiento académico de unos y otros. Estas diferencias socioeconómicas, según Jadue ${ }^{(29)}$, se reflejan en la falta de lugares adecuados de estudio, la carencia de material escolar y la ausencia de apoyo familiar en el estudio, lo que podría explicar en parte los menores resultados académicos obtenidos. Sin embargo, es preciso considerar que los alumnos migrantes con mayor tiempo de residencia en España tienden a mejorar los resultados respecto de los recién llegados ${ }^{(28)}$, hecho que resalta nuevamente la función integradora que desarrolla el entorno escolar. 
En relación a la práctica de $\mathrm{AF}$, se encuentra una menor realización por parte del alumnado migrante, resultados coincidentes con estudios internacionales como el de Williams et $\mathrm{al}^{(30)}$, donde la probabilidad de realizar AF era menor en aquellos adolescentes con progenitores no autóctonos. Analizando las franjas horarias de realización de AF, las diferencias se presentan entre las 18:00 y las 22:00 horas, horario habitual de realización de actividades deportivas organizadas, y en el que se obtiene un porcentaje de práctica muy inferior por parte de los migrantes (48\% por un $75 \%$ de los autóctonos). En este contexto, Steenhuis et al ${ }^{(31)}$ señalan al nivel socioeconómico como un claro predictor en la realización de AF, determinando en gran medida la posibilidad de acceso a estas actividades, y explicando además la renuncia de las familias con menores recursos económicos a la inscripción de sus hijos en las prácticas que tienen un coste económico. Esta menor participación en actividades organizadas tiene influencia sobre los valores globales de AF, los cuales también son inferiores entre los migrantes, hecho que podría asociarse a los menores niveles de $\mathrm{VO}_{2}$ max de estos, ya que la literatura existente asocia ambos parámetros ${ }^{(32)}$.

Todo lo contrario ocurría en horario escolar, donde no se reportan diferencias entre unos y otros. Destaca nuevamente la labor de los centros educativos y, más concretamente, de las clases de Educación Física, en la promoción equitativa de hábitos de salud, ya que un alto porcentaje de la AF diaria realizada por todos los adolescentes se hace efectiva durante la mañana ${ }^{(33)}$.

Respecto a la DM, los resultados obtenidos reflejan una menor adherencia por parte de los adolescentes migrantes. Martínez et $\mathrm{al}^{(34)}$ hallaron que el porcentaje de adolescentes con una adherencia a dietas de calidad baja y baja/media era superior en los migrantes. En la misma línea, en la investigación de Esteban et $\mathrm{al}^{(35)}$, los adolescentes migrantes presentaron una mayor probabilidad de no cumplir las recomendaciones nutricionales en cuanto al consumo de carne, legumbres, pescado, cereales y leche, además de mostrar una mayor ingesta de dulces y productos azucarados. En nuestro estudio, los migrantes presentan un mayor consumo de comida rápida y dulces, además de pasta y arroz, lo que se puede justificar desde el prisma socioeconómico hallado en estudios anteriores ${ }^{(36)}$, ya que son productos más accesibles y económicos. Esta justificación se apoya igualmente en los resultados de regresión, donde la varianza de la adherencia a la DM en función del nivel socioeconómico era explicada en mayor medida en la población migrante $(3,1 \%)$ que en la autóctona $(0,16 \%)$. No obstante, es preciso considerar que la menor adherencia a la DM en los migrantes puede verse influenciada por otros muchos factores como los psicosociales, el entorno, las creencias religiosas o las cuestiones históricas, destacando necesariamente la herencia cultural alimentaria. Esta conformará modelos alimentarios y nutricionales que pueden ser muy dispares a los mediterráneos ${ }^{(37)}$.

Partiendo de los supuestos anteriores, el mayor consumo de dulces y comida rápida, junto con la menor AF realizada por parte de los migrantes, podrían explicar el mayor IMC y los porcentajes de sobrepeso y obesidad reportados por estos. En consecuencia, los porcentajes de insatisfacción corporal también son mayores entre la población migrante, concretamente diez puntos superiores $(28 \%$ por $18,3 \%)$. De este modo, la asociación hallada entre la satisfacción corporal y el bienestar psicológico en la población migrante podría explicar sus menores niveles de autoestima, hecho que genera una mayor probabilidad de padecer trastornos psicológicos como la depresión ${ }^{(38)}$.

La obtención de una muestra representativa en el presente estudio posibilita una descripción minuciosa de la percepción de la salud en 
el alumnado migrante y en el autóctono, desde una perspectiva tanto física como mental y social. Se siguió un protocolo de actuación común en todos los centros, utilizando cuestionarios de fiabilidad y validez demostrada en anteriores estudios con poblaciones del mismo perfil. Sin embargo, el hecho de ser cuestionarios autocumplimentados motiva que su realización esté sometida a la subjetividad, valorando como prospectiva de mejora el uso de instrumentos más objetivos como acelerómetros o registros de frecuencia de consumo diaria. Otra de las limitaciones del estudio fue la indiscriminación de los países de procedencia de los adolescentes migrantes, lo cual hubiese permitido detallar los resultados según la nacionalidad y ayudar a descifrar de una forma más precisa el estado actual. No obstante, esta opción se descartó porque dicho desglose generaba múltiples grupos con un bajo nivel de representatividad. Por otro lado, el diseño transversal de la investigación impide establecer relaciones de causalidad entre las diferentes variables, por lo que la realización de nuevos estudios de carácter longitudinal y de intervención resultan necesarios.

A modo de conclusión, la investigación fue realizada desde un punto de vista integral del estado de salud de los adolescentes, valorando diferentes indicadores de la salud física, psicológica y social, y analizando las diferencias entre la población migrante y autóctona. Los resultados reflejan menores niveles de actividad física, $\mathrm{VO}_{2}$ máx, calidad de vida relacionada con la salud, autoestima, dieta mediterránea, rendimiento académico y nivel socioeconómico en el alumnado migrante. Condicionantes de vida como el nivel socioeconómico resultan claves en la salud de esta población. Teniendo en cuenta que el contexto escolar proporciona un efecto protector sobre algunas de las variables estudiadas, como la práctica de AF y la CVRS, resulta necesario continuar desarrollando líneas de actuación relacionadas con la crea- ción de espacios inclusivos y la interrelación entre las culturas, favoreciendo así la integración y, por tanto, el bienestar del alumnado migrante. Por otro lado, en relación con el entorno extraescolar, se hace necesaria la implementación de estrategias que promuevan el acceso a la práctica física y a alimentos saludables con el objetivo de favorecer la mejora de la salud comunitaria y, en especial, de los grupos más vulnerables.

\section{BIBLIOGRAFÍA}

1. Instituto Nacional de Estadística. Padrón Continuo en España. 1 de enero de 2019.

2. Ronda E, Ortiz G, Hernando C, Vives C, Gil D y Casabona J. Características generales de los artículos originales incluidos en las revisiones bibliográficas sobre salud e inmigración en España. Revista Española de Salud Pública. 2014;88(6):675-685

3. Furumoto A, Gehlert S, Sohmer D, Olopade O y Sacks T. Early-life conditions and mechanisms of population health vulnerabilities. Health Affairs. 2007;26(5):1238-1248.

4. Meroc E, Moreau N, Lebacq T, Dujeu M, Pedroni C, Godin I y Castetbon K. Immigration and adolescent health: the case of a multicultural population. Public health. 2019; 175:120-128.

5. San Mauro I, Megías A, García de Angulo B, Bodega P, Rodríguez P, Grande G, Micó V, Romero E, García N, Fajardo D y Garicano E. Influencia de hábitos saludables en el estado ponderal de niños y adolescentes en edad escolar. Nutrición Hospitalaria. 2015;31(5):1996-2005.

6. Breslow L y Enstrom, JE. Persistence of health habits and their relationship to mortality. Preventive medicine. 1980;9(4):469-483.

7. Burdette AM, Needham BL, Taylor MG y Hill TD. Health lifestyles in adolescence and self-rated health into adulthood. Journal of health and social behavior. 2017;58(4): 520-536. 
8. Vieno A, Santinello M, Lenzi M, Baldassari D y Mirandola M. Health status in immigrants and native early adolescents in Italy. Journal of community health. 2009;34(3):181-187.

9. Imhof K, Faude O, Donath L, Bean-Eisenhut S, Hanssen H y Zahner L. The association of socio-economic factors with physical fitness and activity behaviours, spinal posture and retinal vessel parameters in first graders in urban Switzerland. Journal of sports sciences. 2016;34(13):1271-1280.

10. Fernández-Alvira JM, Börnhorst C, Bammann K, Gwozdz W, Krogh V, Hebestreit A, Barba G, Reisch L, Eiben G, Iglesia I, Veidebaum T, Kourides Y, Kovacs E, Huybrechts I, Piegot I y Moreno LA. Prospective associations between socioeconomic status and dietary patterns in European children: the Identification and Prevention of Dietary-and Lifestyleinduced Health Effects in Children and Infants (IDEFICS) Study. British Journal of Nutrition. 2015;113(3):517-525.

11. Silva M, Loureiro A y Cardoso G. Social determinants of mental health: a review of the evidence. The European Journal of Psychiatry. 2016;30(4):259-292.

12. Constant AF. The healthy immigrant paradox and health convergence. DICE Report. 2017;15(3):20-25.

13. Horenczyk G y Tatar M. Conceptualizing the school acculturative context: School, classroom, and the immigrant student. Realizing the potential of immigrant youth. 2012:359-375.

14. Atienza FL, Moreno Y, Balaguer I. Análisis de la dimensionalidad de la Escala de Autoestima de Rosenberg en una muestra de adolescentes valencianos. Revista de Psicología Universitas Tarraconensis. 2000;22:29-42.

15. Aymerich M, Berra S, Guillamón I, Herdman M, Alonso J, Ravens U y Rajmil L. Desarrollo de la versión en español del KIDSCREEN: un cuestionario de calidad de vida para la población infantil y adolescente. Gaceta Sanitaria. 2005;19(2):93-102.
16. Stunkard A y Stellar E. Eating and its disorders. En: Cash T, Pruzinsky T. Body Images, Nueva York. Guilford Press; 1990: p. 3-20.

17. Marrodan V, Montero MS, Mesa JL, Pacheco M, Gonzalez I, Bejerano I, Lomaglio DB, Verón JA y Carmenate M. Realidad, percepción y atractivo de la imagen corporal: condicionantes biológicos y socioculturales. Zainak, Cuadernos de Antropología-Etnografía. 2008; 30:15-28.

18. Serra L, Ribas L, Ngo J, Ortega RM, García A, Pérez C y Aranceta J. Food, youth and the Mediterranean diet in Spain. Development of KIDMED, Mediterranean Diet Quality Index in children and adolescents. Public health nutrition. 2004;7(7): 931-935.

19. Martínez D, Martínez de Haro V, Pozo T, Welk GJ, Villagra A, Calle ME, Marcos A y Veiga OL. Fiabilidad y validez del cuestionario de actividad física PAQ-A en adolescentes españoles. Revista española de salud pública. 2009; 83: 427-439.

20. Fonseca E, Paíno M, Lemos S, Villazón Ú, Muñiz J. Validation of the Schizotypal Personality Questionnaire Brief form in adolescents. Schizophrenia Research. 2009; 111(1-3): 53-60.

21. Leger LA, Mercier D, Gadoury C, Lambert J. The multistage 20 metre shuttle run test for aerobic fitness. Journal of sports sciences. 1988; 6(2): 93-101.

22. Onis MD, Onyango AW, Borghi E, Siyam A, Nishida C, Siekmann J. Development of a WHO growth reference for school-aged children and adolescents. Bulletin of the World health Organization. 2007;85:660-667.

23. Hartley, JEK, Levin K y Currie C. A new version of the HBSC Family Affluence Scale-FAS III: Scottish Qualitative Findings from the International FAS Development Study. Child Indic. Res. 2016;9:233-245.

24. Castañeda H, Holmes SM, Madrigal DS, Young MED, Beyeler N y Quesada J. Immigration as a social determinant of health. Annual review of public health. 2015;36:375-392. 
25. Zlobina A y Páez D. Aculturación y comunicación intercultural: El caso de inmigración en España”. En: Cabecinhas R y Cunha L. Comunicación Intercultural. Perspectivas, dilemas y desafíos. Famalicão: Edições Húmus; 2017: p. 37-59.

26. Hjalmarsson S y Mood C. Do poorer youth have fewer friends? The role of household and child economic resources in adolescent school-class friendships. Children and Youth Services Review. 2015; 57:201-211.

27. Boletín Oficial del Estado. Ley Orgánica 8/2013, de 9 de diciembre, para la mejora de la calidad educativa. BOE núm. 295 de 10/12/2013.

28. Zinovyeva N, Felgueroso F y Vázquez P. Inmigración y resultados educativos en España. Boldrin, M., Efectos económicos de la Inmigración en España. Jornadas sobre inmigración. I Informe FEDEA. Madrid. Marcial Pons. 2009: p. 139-178.

29. Jadue G. (1996). Características familiares de los hogares pobres que contribuyen al bajo rendimiento o al fracaso escolar de los niños. Revista de Psicología. 1996;14(1):35-45.

30. Williams W, Li K, Haynie D y Simons-Morton B. Physical activity and sedentary behavior of US immigrant versus non-immigrant adolescents: findings from the NEXT Generation Health Study data. Ethnicity \& health. 2018;23(3):329-338.

31. Steenhuis IH, Nooy SB, Moes MJ y Schuit AJ. Financial barriers and pricing strategies related to participation in sports activities: the perceptions of people of low income. Journal of Physical Activity and Health. 2009;6(6):716-721
32. Mcmurray RG, Harrell JS, Bangdiwala SI y $\mathrm{Hu}$ J.Tracking of physical activity and aerobic power from childhood through adolescence. Medicine and science in sports and exercise. 2003;35(11):1914-1922.

33. Mota J, Santos P, Guerra S, Ribeiro JC y Duarte JA. Patterns of daily physical activity during school days in children and adolescents. American Journal of Human Biology. 2003;15(4):547-553.

34. Martínez CP, Del Olmo RF y Anuncibay J. Evaluación de la calidad de la dieta y su relación con el estatus nutricional en niños y adolescentes de 9 a 15 años de la ciudad de Madrid. Antropo. 2007;14: 60-73.

35. Esteban L, Veiga OL, Gómez S, Regidor E, Martínez D, Marcos A y Calle ME. Adherence to dietary recommendations among Spanish and immigrant adolescents living in Spain; the AFINOS study. Nutricion hospitalaria. 2013; 28(6): 1926-1936.

36. Schröder H, Gomez SF, Ribas L, Pérez C, Bawaked RA, Fíto M y Serra-Majem L. Monetary diet cost, diet quality, and parental socioeconomic status in Spanish youth. PloS one. 2016;11(9): e0161422.

37. Gedrich K. Determinants of nutritional behaviour: a multitude of levers for successful intervention? Appetite. 2003;41(3):231-238.

38. Ozmen D, Ozmen E, Ergin D, Cetinkaya AC, Sen N, Dundar PE y Taskin EO. The association of self-esteem, depression and body satisfaction with obesity among Turkish adolescents. BMC Public Health. 2007;7(1):80. 